\title{
Development of a cold plug valve with fluoride salt
}

\author{
Julien Giraud $^{2}$, Veronique Ghetta ${ }^{2, *}$, Pablo Rubiolo ${ }^{3}$, and Mauricio Tano Retamales ${ }^{1}$ \\ 1 Univ. Grenoble Alpes, LPSC-IN2P3, 38026 Grenoble Cedex, France \\ 2 CNRS, LPSC-IN2P3, 38026 Grenoble Cedex, France \\ ${ }^{3}$ Grenoble INP (Institute of Engineering University Grenoble Alpes), LPSC-IN2P3, 38026 Grenoble Cedex, France
}

Received: 15 February 2019 / Received in final form: 21 June 2019 / Accepted: 27 June 2019

\begin{abstract}
Experimental studies have been developed on a new freeze plug concept for safety valves in facilities using molten salt. They are designed to allow the closure of an upstream circuit by solidifying the molten salt in a section of the device and to passively melt in case of a loss of electric power, thus releasing the upper fluid. The working principle of these cold plug designs relies on the control of the heat transfer balance inside the device, which determines whether the salt inside the cold plug solidifies or melts. The device is mainly composed of steel masses that are dimensioned to provide sufficient thermal heat storage to melt the salt and thus open the cold plug after the electric power is stopped. The final goal of the work is to provide useful recommendations and guidelines for the design of a cold plug for the emergency draining system of a molten salt reactor. Some numerical thermal simulations were performed with ANSYS mechanical (Finite Element Method) to be compared with results of the experiments and to make extrapolations for a new component to be used in a reactor.
\end{abstract}

\section{Introduction}

Molten Salt Reactors have attracted increased attention in recent years because of the design and safety possibilities offered by the use of a liquid fuel. Investigations on such concepts, very different from those of solid combustible reactors, are nowadays based on numerical models whereas experimental knowledge is concentrated on past studies in Oak Ridge National Laboratory in the framework of the MSRE experiment [1,2]. The newly considered concepts focus on fast and thermal spectrum reactors as for example the MSFR concept [3] supported by the European project SAMOFAR (2015-2019). From a safety point of view, two important systems in this reactor are the fuel salt draining system and the Decay Heat Removal system. The first one is used to transfer the fuel salt from the core cavity to dedicated tanks were the salt can be cool-down while keeping the reactivity of the fuel below acceptable margins. Different types of devices can be envisioned to accomplish the function of controlling the molten fuel salt draining from the core cavity to the draining tank.

To test the performance of any component important to the nuclear reactor safety, in particular in order to make meaningful design, it is essential to master all potential physical phenomena and to be able to give valid numerical representation.

\footnotetext{
* e-mail: ghetta@lpsc.in2p3.fr
}

Shut-off valves are designed to be capable of providing very low leakage when closed. In mechanical valves good sealing is provided by metal to metal contact and two types of failure risks exist: self-welding due to the excellent fluxing agents properties of molten fluorides can cause the valve to fail to open; local scratching due to local welding or presence of partial frozen salt can cause the mechanical valve to leak. In case of low speed flow and a role focused on the opening of the flow circuit, the use of a freeze valve can be a reliable alternative solution in applications for passive emergency systems.

We have developed small experimental facilities with inactive salt to investigate the performance and working mechanism of a safety freeze valve, named "cold plug device". The work was performed in two experimental facilities named FFFER (Forced Fluoride Flow for Experimental Research) and SWATH (Salt at Wall: Thermal excHanges). In the former (Sect. 3), a preliminary design of passive cold plug has been tested. Further studies have been carried out in the SWATH set-up with a different geometry (Sect. 4). In Section 5, we extrapolate these results to reactor case.

\section{Concept of cold plug valve with fluoride salt}

Local freezing of certain quantity of liquid can do effective sealing of a pipe, whatever is that liquid, metal or salt. Design of seal shut-off valve is highly dependent on the 
liquid and solid physical properties. Basically, a cold plug valve is a metallic device installed in a pipes network, equipped with heating and cooling system able to crystallize and melt a portion of the inside coolant. The objective is to prevent coolant from flowing from one side to the other or to isolate different parts of a system. In salt case, the crystallized part itself is not able to allow gas tightness, due to the random contact of the solidified salt on the metallic wall, possibility of porosity and cracks. The plug remains gas tight only as long as some liquid is left upstream. Of course, this type of valve cannot interrupt an established flow due to the high calorific capacity and low conductivity of molten salts. Solidification of the salt can takes place even in presence of flow convection but it would require a very efficient cooling system able to compensate for the heat energy provided by the flowing salt. Reliable closure of a cold plug requires then that the liquid salt in the cold plug area and in the upstream and downstream areas remain almost still.

Test of three different types of frozen-seal "valves" have been carried out during the MSRE reactor studies at the Oak Ridge National Laboratory [1,2]. These studies performing the proof of concept of the use of freeze valves to establish shutoff in small diameter lines in a static salt system. Such valves were then used in the MSRE experiment with a $\mathrm{LiF}-\mathrm{BeF}_{2}-\mathrm{ZrF}_{4}$ (melting temperature $=$ $434^{\circ} \mathrm{C}$ ) as carrier salt. The melting temperature of the molten salt fuel mixture was $434^{\circ} \mathrm{C}$. The systems includes heating and cooling systems used to maintain the crystallized area in condition for rapid melting when the cooling is stopped, that is to say: the opening mode of the plug valve was to keep the heating and cut the cooling ("frozen mode" [2]).

While the operation of these valves during MSRE experiments was considered as a success, one drawback of this cold plug design was that in the event of a heaters power failure, the plug does not melt. We preferred to thermally design the cold plug such as the system opens, after the cooling and heating systems are both simultaneously stopped, by the heating effect arising from the redistribution of thermal energy stored in the cold plug structure.

\section{Cold plug study in the FFFER facility}

The FFFER experiment is a project started in 2009 year whose acronym means: Forced Fluoride Flow for Experimental Research. A cold plug design was firstly implemented in this experiment as it is described in this section. The FFFER facility is a $\mathrm{LiF}-\mathrm{NaF}-\mathrm{KF}$ eutectic (FLiNaK) salt forced convection loop whose main objectives were: (a) study the liquid/gas separation in the case of a continuous on-line molten salt cleaning process and (b) acquire technical experience in designing and operating high temperature salt experiments. The first objective is related to critical MSFR design aspects since in a molten salt reactor liquid/gas separation can be used to extract part of the fission products generated by the reactor in the fuel molten salt. Actually, on-line bubbling is an efficient process to capture dispersed particles in a liquid and partly

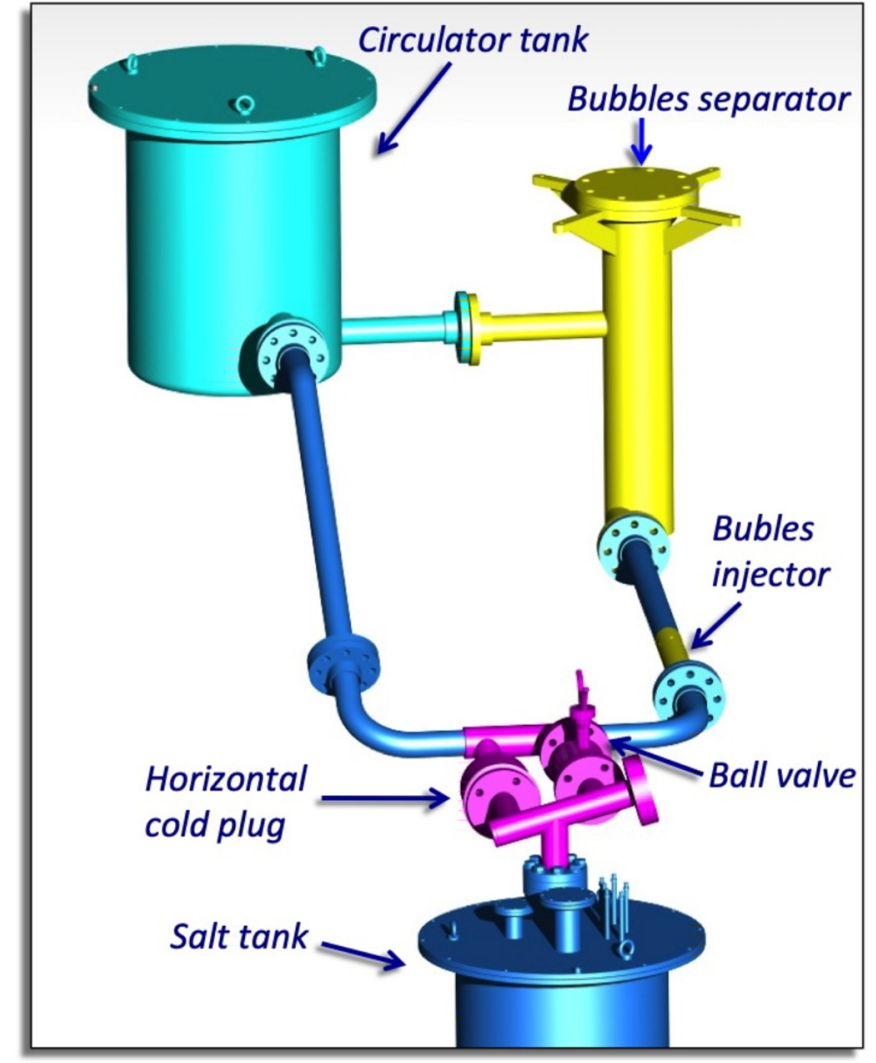

Fig. 1. Global design of the FFFER loop.

absorb dissolved gas. It consists in injecting bubbles into the salt while it is circulating in the pipes. The experimental work undertaken in the laboratory focused on the process itself, from bubble injection to liquid/gas separation. The pipes diameter used in that facility was quite large ( $57 \mathrm{~mm}$ inner diameter) to obtain a representative bi-phase flow, with negligible disturbing effects due to the walls. As can be seen in Figure 1, the main components of the FFFER loop was the molten salt circulator, the bubble separator device, the salt tank, the gas injectors (argon), the loop pipes and a double valve system for salt tank/loop separation. The double valve system is constituted of a ball valve and a cold plug device designed so as to open in case of incident to flush the salt into the tank. For instance, if the circulator and the heating systems stop, following an electric failure or any other incident, the calorific capacity of the salt (whose nominal temperature is about $600^{\circ} \mathrm{C}$ ) provides about 15 to 20 min before freezing in coldest area of the circuit.

About $50 \mathrm{~L}$ of molten FLiNaK have to be drained, from the circuit towards the salt tank, within this time lapse. To prevent the risk of ball valve jamming in the closed position, a cold plug device was the designed as safety device, to passively melt in case of an excessive temperature rise or a loss of electric power. The working principle of the cold plug relies on the passive control of the heat transfer balance inside the device, which determines whether the salt inside the cold plug solidifies or melts. Likewise the MSRE cold plug, the cold plug device in 

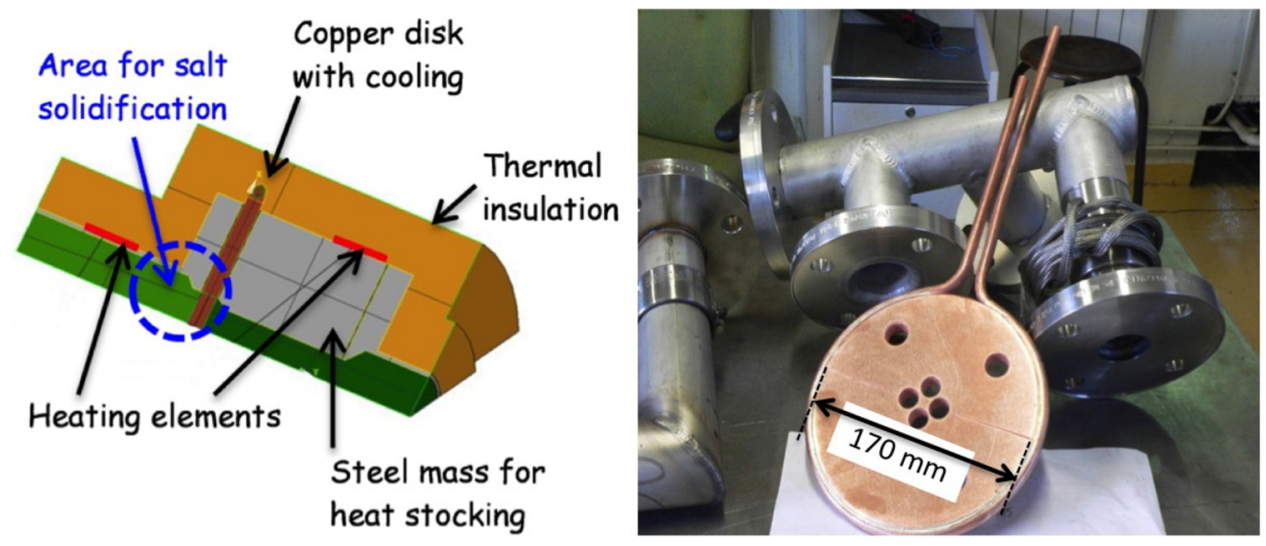

Fig. 2. Design of the first cold plug used during FFFER experiments and details of the cooling copper piece before mounting and tests.

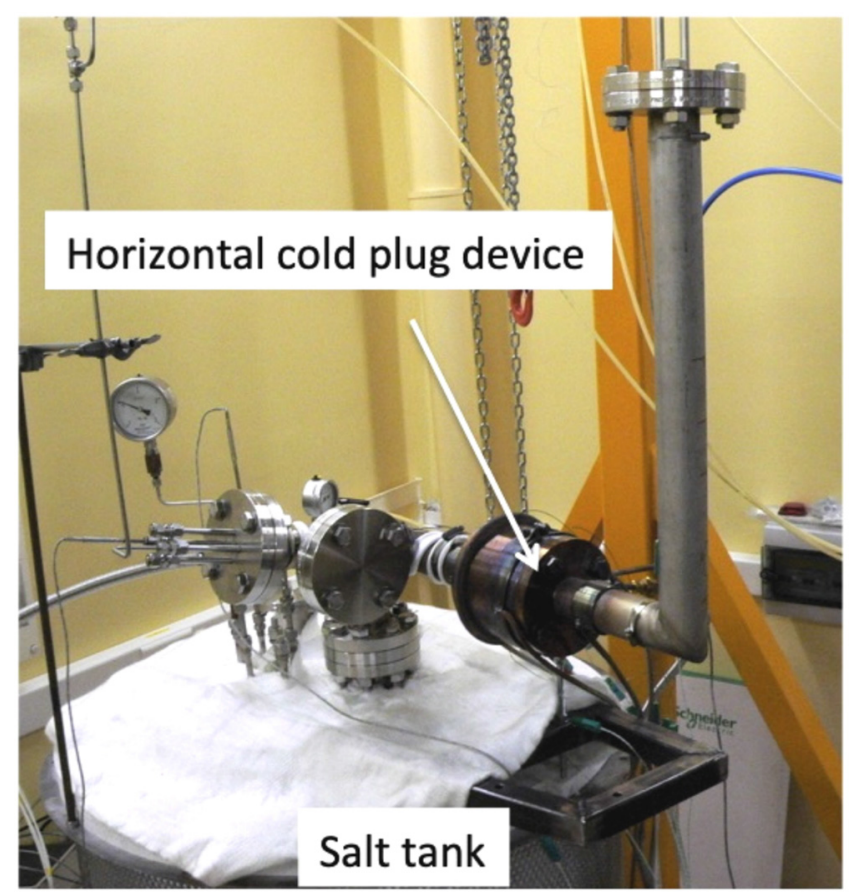

Fig. 3. Configuration of the first tests with a simple vertical pipe instead of the loop circuit.

FFFER is both heated and cooled but one important improvement with respect to the MSRE concept is that we increase the structural steel mass to store thermal energy that will increase the energy transfer to the cold plug after the cooling and heating have stopped as seen in Figure 2 (left part). The quantity of thermal energy stored in this steel mass has to be sufficient to initiate the melting of the borders of the cold plug and thus initiating the flow. Then, the energy brought by the hotter molten salt existing in the upper part of the device salt will quickly melt the remaining solid plug.

When cooling and heating are stopped (e.g., due to a loss of electrical power in FFFER), the thermal energy stored in the steel mass is transferred by conduction to the solidified salt region causing its melting. The main difficulty of the cold plug concept design resides in designing the shape of the walls in the crystallization area in order to manage sufficient heat transfer (and thus reducing the melting time). In the steel mass, the internal diameter is slightly restricted (34 $\mathrm{mm}$ in diameter) with regard to the pipes diameter $(57 \mathrm{~mm}$ in diameter $)$. We use a copper disk (see Fig. 2, left part) between two steel masses to ease the plug formation, conservation and melting. It improves the energy transfer from the steel heat storing mass to the cold plug during the stage of melting, when the system is delivered to itself and with no heating nor cooling anymore. Thermocouples are inserted in grooves between the copper disk and steel mass in the area for salt solidification.

In the FFFER experiment the cold plug is placed with a horizontal orientation as can be seen in Figure 1. The first tests of this component have been done before the FFFER loop achievement with only a vertical pipe connected with the salt tank to perform them (Fig. 3). The different stages for cold plug formation are detailed in Figure 4. First, the salt tank is pressurized in order to push upward the molten salt inside the pipe of the vertical test section. Then, the pressure difference between the tank and the cover gas of the test section is set to a value that maintains the salt level in the vertical section and allows to cold down a pipe section to form the cold plug. After some time in this condition, the tank pressure is released and gas injected in the pipe between the tank and the plug in order to drain the molten salt left below the plug to the tank. The pressure of the cover gas in the vertical section is then increased $(\mathrm{P} 3>\mathrm{P} 2)$ in order to verify the tightness of the salt plug (no leaks). Detection of a leak is done both by the measurement of the salt level in the pipe and the pressure evolution. The cold plug is maintained closed thanks to equilibrium between heating and cooling. After a first rough numerical simulation these stability conditions have been determined by trial and error.

The local temperature of the solidified area is kept about $20-25^{\circ} \mathrm{C}$ lower than the salt melting temperature.

Test of the time to opening is done by suppression of heating and cooling of the plug. In normal conditions, the temperature of the parts of the disk under mechanical stress (between steel masses) remains lower than $500{ }^{\circ} \mathrm{C}$. 


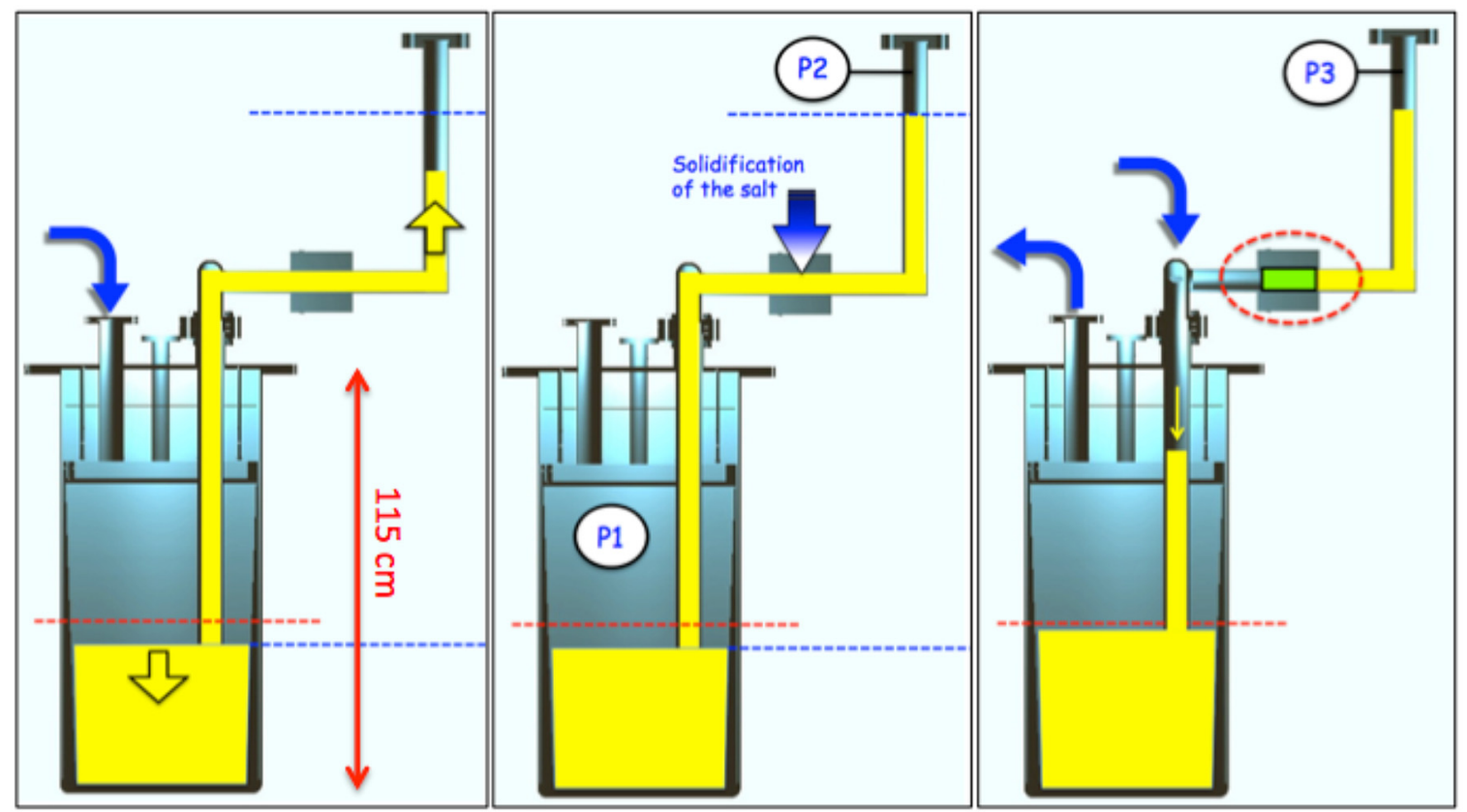

Fig. 4. Sequences of cold plug formation in the simplified configuration, left: rise of the molten salt in the pipe above the plug, middle: salt level kept constant and cooling of the plug, right: release of tank pressure and gas injection to drain the molten salt left below the plug.

During the draining of the salt, the central part of the disk in contact with the flowing salt withstand for a moment higher temperature (until $600{ }^{\circ} \mathrm{C}$, temperature of the flowing salt) but without mechanical constraint.

Figure 5 presents an example of the temperature measurements obtained during a cold plug opening test. The 0 time corresponds to the cooling and heating cut off, the green line presents the temperature evolution of the horizontal pipe before the cold plug. After the shut down, temperature decrease regularly, the vertical dotted line mark out the moment where this regular decreasing stop because of a small flow coming from the upper part of the set up, this is the clear indication that the plug begin to open. The flow is at first very small and has no effect on the temperature of the downstream part (red curve). Then the plug opening is going on essentially owing the additional energy brought by the flowing liquid. The time lapses for the opening the plug in these simplified conditions (only a small vertical tube and no loop) was compatible with the FFFER loop requirements.

Several tests and modifications were done before mounting the various components of the final loop configuration. Figure 6 shows the new static configuration always with a vertical pipe but with the ball valve mounted in parallel, and Figure 7 shows the final assembly in flowing loop configuration. During the subsequent experiments performed in the FFFER loop, we have noted that performance and the working points of the cold plug in its final configuration were considerably different from those measured during the preliminary tests. We attribute these differences to a flow recirculation in the cold plug connecting pipe (see Fig. 7) that appears as results of the molten salt flow in the loop and the temperature gradient.

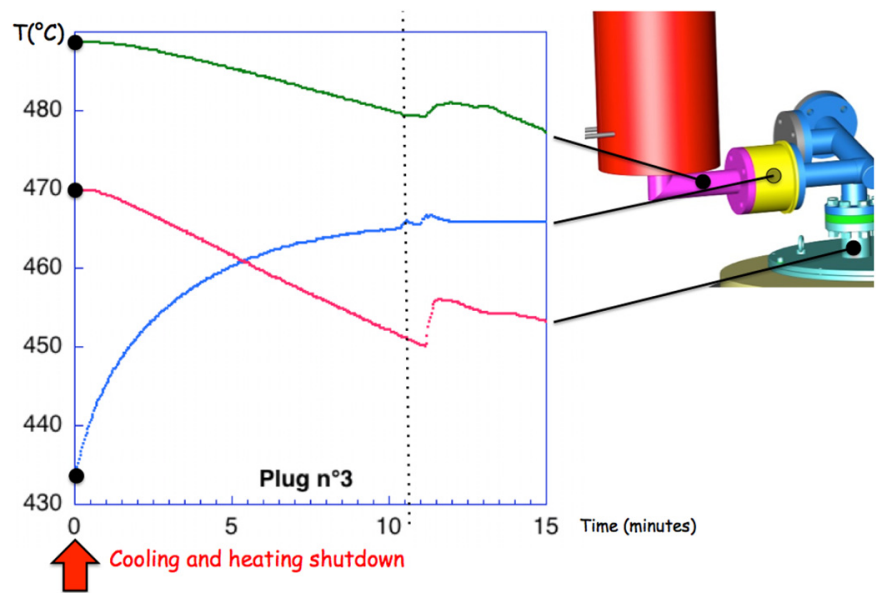

Fig. 5. Example of evolution of local temperatures during a cold plug opening.

This unwanted flow recirculation in the connecting pipe could transfer large heat quantities towards the plug and alter the heat balance of the cold plug.

These changes required some modifications in the cold plug operating parameters (air cooling flow and steel mass heating) but were not out of the reach. Other parameters change (e.g., cold plug distance from loop or the design of the copper disk) needed modifications in the geometrical design and further work on the loop have seen the cold plug dismounting for more simple work, the objectives and difficulties being focused on other parts of the loop. The experience gained from the semi-empirical design of the FFFER "cold plug" device showed the importance of 


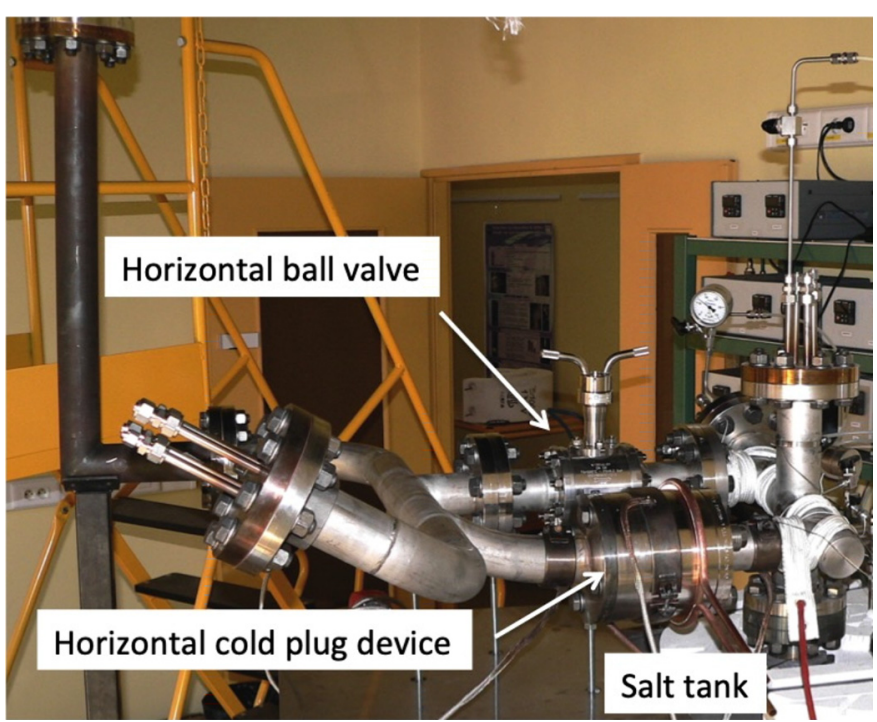

Fig. 6. Second configuration for test with the ball valve mounted in parallel.

developing more accurate tools. These tools are needed to describe in details on the one hand the working of such cold plug and on the other hand the effect of potential flow recirculations close to the cold plug. The later, requires the validation of Computational Fluid Dynamics (CFD) models on experiments dedicated to measure weak thermal effects since few degrees can do the difference between a stable closed plug, an involuntarily opening plug, or a closed locked plug. The salt flows in this type of experiments have also to be precisely controlled or at least known in order to determine their additional thermal contribution. In Section 4, we present a device developed with the purpose to study the operation of a cold plug with the less as possible convective perturbations.

\section{Cold plug study in the swath facility}

Building from the experience gained from the cold plug used in the FFFER loop, and to further improve the understanding of the design of this device, experimental studies have been carried out in a facility called SWATH (Salt at Wall: Thermal ExcHanges) developed in the framework of the European Project SAMOFAR at LPSCGrenoble. The objective of the SWATH experiments is to improve molten salt numerical models used for design and safety studies [4], and more specifically during MSR fuel salt draining [5]. The fluoride salt used is the same as in the FFFER experiments: the $\mathrm{LiF}-\mathrm{NaF}-\mathrm{KF}$ eutectic mixture and argon is used as cover gas.

The operation of SWATH facility is based on a discontinuous working principle regulating the pressure difference between two tanks. For thermal studies on flowing salt, the flow is established in a channel section between both tanks. The pressure control system is designed to generate a stable flow in the studied section during the operation. Valves are present in the circuit that can close the salt circulation and allow the use of the tanks separately. The cold plug studied in SWATH project is

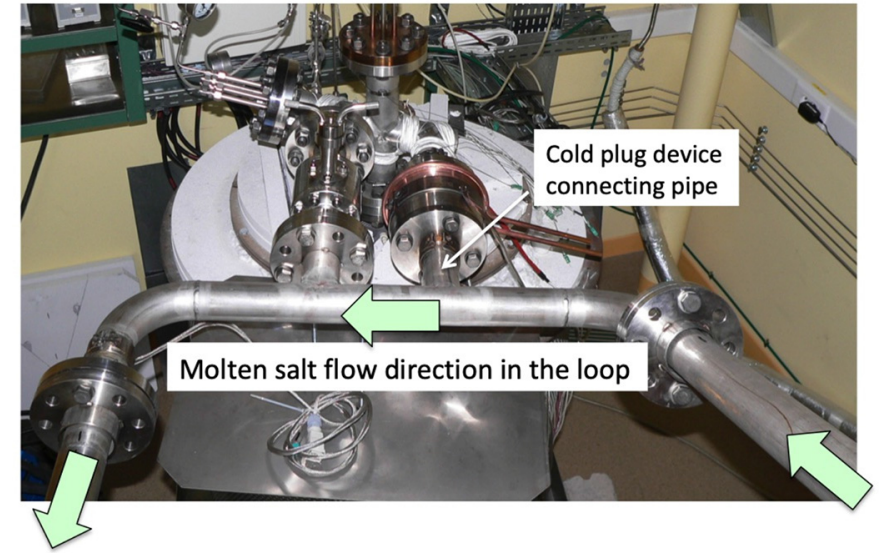

Fig. 7. Final assembly of the twofold valve system between the tank and the loop. The photograph has been taken before the whole insulation implementation.

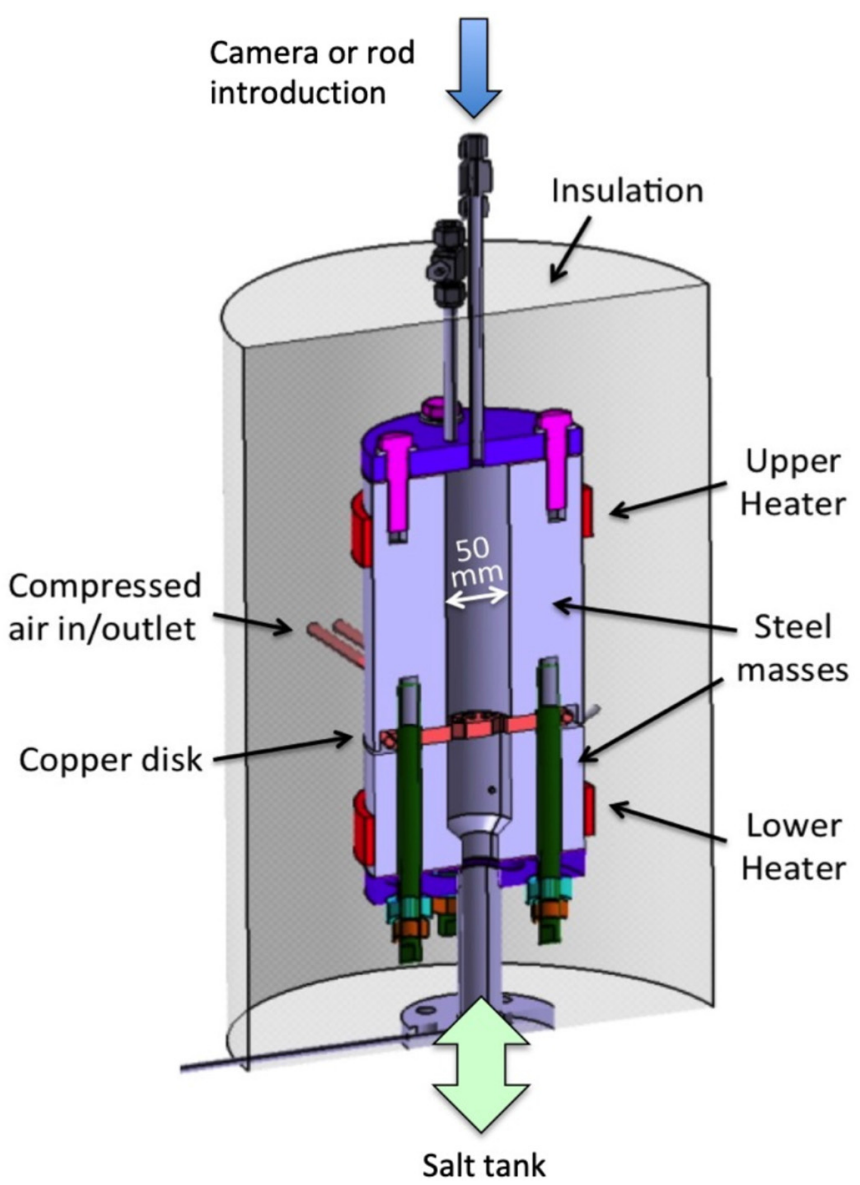

Fig. 8. Layout of the cold plug device tested in SWATH facility.

located vertically above the downstream tank (Figs. 8 and 10) and can be used separately, without flowing salt in the other parts of the SWATH facility. The pressure control of this tank allows the rise of the salt in the cold plug, and makes also possible to keep the position of the liquid/gas interface at a constant level. 


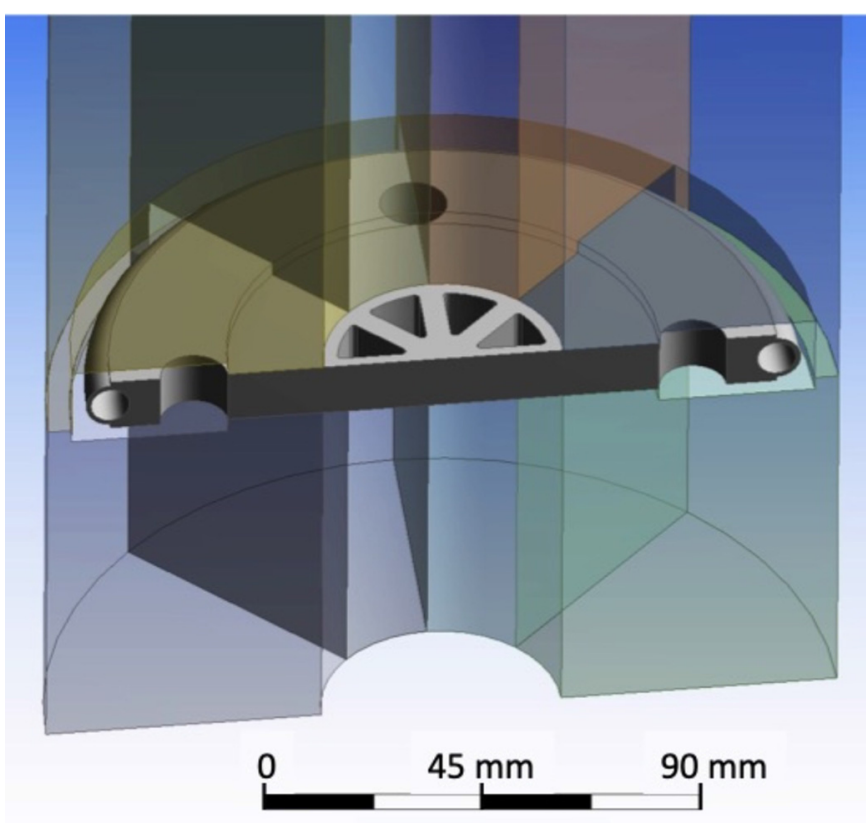

Fig. 9. New copper disk feature.

As the principal aim is focused on the understanding of the cold plug working processes, we chose to mount it vertically above the downstream tank of SWATH set-up. This vertical geometry is expected to allow for a better control and measurements of the solidification/melting processes that take place during the cold plug operation. In SWATH, the cold plug device is studied alone, with no external salt flow to have a better control of the heat balance in the device. This vertical arrangement simplifies the data collection, the experiment operation and also provides a simpler access to the upper part of the solidified area. The salt level reached during the filling of the cold plug cavity is measured by a stainless steel rod, used as a contactor, which is inserted inside the cold plug device (see Fig. 8). The position of this contactor is adjustable. A mini-camera can also be inserted in the same way to inspect the cavity after cooling of the whole system.

The cooling is provided by airflow inside the copper disk of the device. The design presented in Figure 8 has been improved from FFFER experiments notably with new feature for the copper disk (Fig. 9): petal shape for the middle of the disk and whole inclusion of the disk inside the steel masses. The petal shape increases the heat transfer from the steel masses towards the solidified salt. On the other hand, the complete insertion of the disc into the steel masses improves the cooling during the plug formation phase and during normal operation (to avoid unwanted openings). The volume of the lower steel mass has also been increased to increase the heat capacity.

Figure 10 shows the cold plug device before thermal insulation.

The running sequence includes the stages implemented in the order given below:

- a: thermal equilibrium of the steel masses at about melting temperature of the salt;

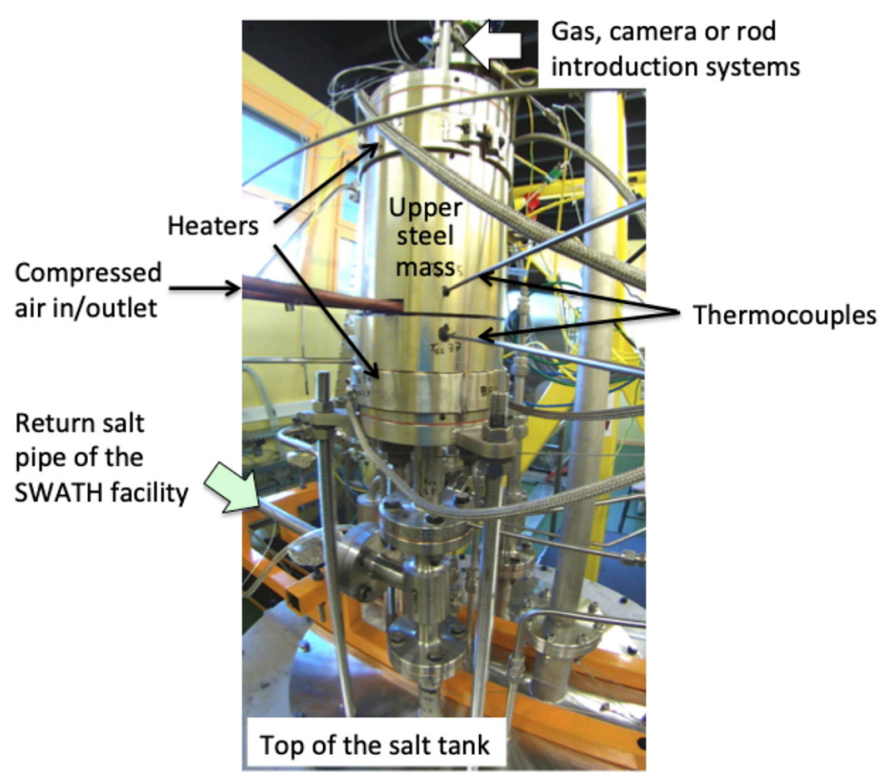

Fig. 10. Cold plug device in the SWATH facility, picture taken before thermal insulation of the system.

- b: filling of the cold plug area;

- c: starting-up of the cooling and keeping the liquid salt at constant level during solidification. This stage takes about $10 \mathrm{~min}$ depending on cooling and heating conditions. The size of the crystallized volume depends on the heating and cooling parameters chosen, it determines partly the stabilization time of the stage (d);

- d: draining of the liquid salt in the downstream part;

- e: stabilization time which can be long due to thermal inertia of the system (about $5 \mathrm{~h}$ ). The normal operation settings (heater temperature and air cooling flow rate) are different from that of the formation stage. They determine the size of the frozen salt plug and have then a direct influence on the plug opening time;

- f: cold plug opening, pressure above the cold plug was increased in several steps to check whether it induced the opening of the plug valve. Liquid level measurements were also done to verify that no liquid leak had been taken place.

Cold plug thermal simulations were performed with ANSYS mechanical (Finite Element Method) only on the cold plug device, but taking into account all its components and insulation with convective and radiant thermal transfers at the interface between atmosphere and insulation. The cooling system is described with convective coefficient to represent the exchanges between compressed air and the copper circuit. Possible thermal convection in the liquid salt is not considered and the numerical simulation is only a thermal one with an enthalpy-based method using an apparent heat capacity. The enthalpy curve was used to take into account the phase change energy in the numerical model employed in the transient simulation of cold plug opening step.

Data used for the numerical simulation are gathered in Table 1. The parameters for the experiments and for the numerical simulations are the compressed air volumetric 
Table 1. Data used for the SWATH cold plug simulations.

\begin{tabular}{lllll}
\hline & Temperature & 304L stainless steel & Copper & LiF-NaF-KF \\
\hline Thermal conductivity $\left(\mathrm{W} \cdot \mathrm{m}^{-1} \cdot \mathrm{K}^{-1}\right)$ & 500 & 20.5 & 334 & 0.6 \\
Density $\left(\mathrm{Kg} \cdot \mathrm{m}^{-3}\right)$ & 500 & 7900 & 8933 & 2113 \\
Heat capacity $\left(\mathrm{J} \cdot \mathrm{Kg}^{-1} \cdot \mathrm{K}^{-1}\right)$ & 500 & 556 & 1000 & 1798 \\
Latent heat $\left(\mathrm{J} \cdot \mathrm{Kg}^{-1}\right)$ & & & 1085 & $4.03 \times 10^{5}$ \\
Melting temperature $\left({ }^{\circ} \mathrm{C}\right)$ & & 1400 & 462 \\
\hline
\end{tabular}

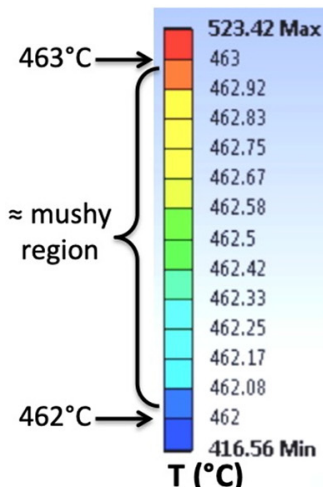

Copper disk thickness
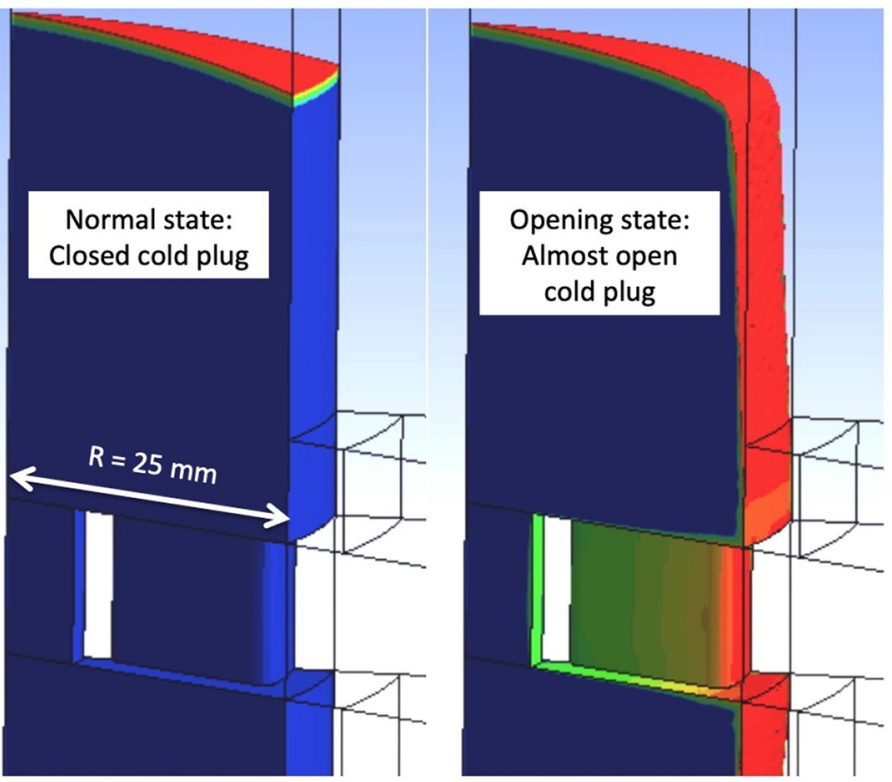

Fig. 11. Energetic numerical ANSYS simulations of the cold plug experiment in SWATH. The left figure corresponds to the "zero time", the instant of the cooling and heating shutdown. The right figure is for the instant where the liquid salt find a path against the wall $(t=378 \mathrm{~s})$. With the temperature color scale used, only the solid salt and a thin mushy region represented with a fine color scale varying with temperature are shown, the liquid covering salt does not appear in the figure.

flow rate in the cooling plate and the temperature set up by the heating elements on the steel structure.

Our simulations are not precise to describe the bottom size of the cold plug (solid salt in contact with Argon gas) but can provide an adequate description of the cold plug opening process. An example of numerical simulation results is presented in Figure 11 with two stages of the cold plug "life": the normal state just before stopping cooling and heating (close valve) and the state where the plug is almost open, i.e. when a continuous path of liquid salt appears against the wall. Using the parameters of the corresponding experiment (upper heater temperature: $550^{\circ} \mathrm{C}$, lower heater temperature: $510^{\circ} \mathrm{C}$, cooling gas flow rate $4.5 \mathrm{~m}^{3} \cdot \mathrm{h}^{-1}$ ), the numerical simulation result places this moment at $378 \mathrm{~s}$. With the temperature color scale used, only the solid salt and a thin mushy region represented with a fine color scale varying with temperature are shown, the liquid covering salt does not appear in the figure.

The salt melting begins along all the wall of the steel mass and copper holes. This moment is considered as the "opening time". This is the instant when the liquid salt finds a flow path along the steel and copper walls. At this stage, only a very small part of the plug is melted, but as long as the temperature of the upstream liquid salt is sufficient, wider opening of the plug can occur. The total energy required to completely open the plug is not provided by the device itself but by the flow of the upstream salt. Since the trigger phenomenon of the opening takes place at the lateral interfaces, the total volume of the normal crystallized plug is not a relevant condition for cold plug melting.

The vertical orientation makes possible measurements of the solid salt thickness above the copper disk during experiment when plug is closed and steady state thermal conditions reached. The measuring technique uses the contactor rod (see Fig. 8), already used for liquid salt level measurement, as a depth gauge that is brought into contact with the surface of the solid salt. The thickness of the solid is estimated with regard to a mechanical guide mark. There is a good agreement between the thickness of the simulated cold plug and the experimental thickness measured. For example, in the previous case (with the following experimental parameters: upper heater temperature: $550{ }^{\circ} \mathrm{C}$, lower heater temperature: $510^{\circ} \mathrm{C}$, cooling gas flow rate $4.5 \mathrm{~m}^{3} \cdot \mathrm{h}^{-1}$ ), which opens at $378 \mathrm{~s}$ according to the 


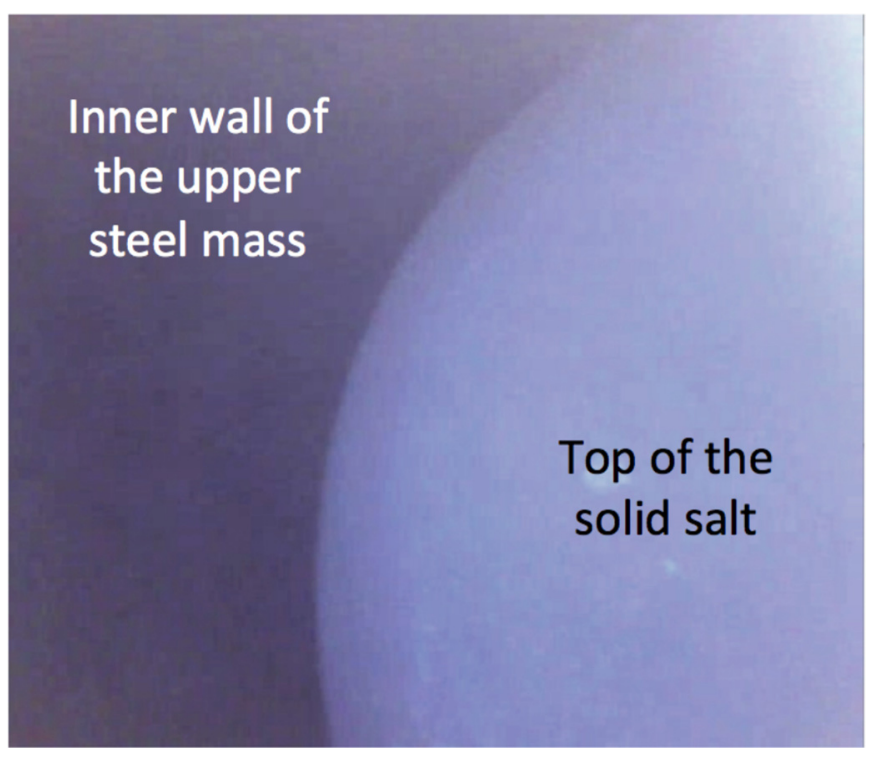

Fig. 12. Picture taken inside above the residual salt after cooling of the whole system.

numerical simulation, the solid thickness provided by the numerical simulation is $38 \mathrm{~mm}$ to be compared to the measured one: $39.5 \mathrm{~mm}$.

As in our experiments the quantity of liquid salt stored in upper part of the device is not sufficient to induce the complete melting of the plug, the shape of the residual solid can be verified by introduction of a small camera after cooling of the whole assembly. The way in for the camera is specified in Figures 8 and 10. Figure 12 shows a picture taken above the residual salt inside the steel mass.

Figure 13 shows the temperature data obtained during the same previous cold plug experiment from four thermocouples inserted in steel masses very near to the copper disk ( $8 \mathrm{~mm}$ abovefor Th1 and Th3,20 mm below for Th2 and Th4). These measurements provide information on the kinetics of the opening process, in order to compare simulated opening time with detected opening time. Cover gas pressure is also measured in the upper part of the cold plug.

At the beginning of the test, the pressure in the upper part was increased for a last time (blue curve) and the cooling and heating power of the cold plug were stopped. The "time zero" is identified by the change on the value of a thermocouple located at the outflow of the cooling gas. Temperatures of the thermocouples situated above the copper disk (Th1, Th3) were slightly superior to those of the Th2, Th4 situated under the disk. After stopping the power, all temperatures increased regularly as transfer of the steel mass thermal energy occurs in this area, which was then not cooled any more. Roughness observed in the curves in Figure 13 is an artifact due to thermocouples measurement noise. In SWATH cold plug experiments temperature readings are not used for opening detection but rather for comparison with numerical simulations of the cold plug opening. A clear drop of the upper vessel pressure (dotted blue circle) indicates the beginning of plug opening.

Opening time of $380 \mathrm{~s}$, as shown in the Figure 13, can be obtained with the present design of cold plug.

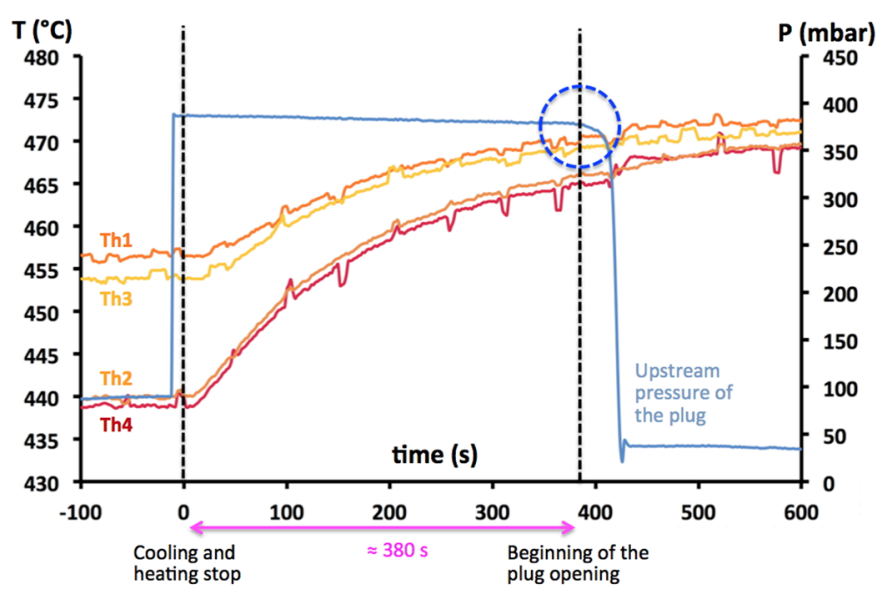

Fig. 13. Temperature and pressure measurements obtained from the cold plug device opening stage experiment. The four thermocouples are inserted in steel masses very near to the copper disk ( $8 \mathrm{~mm}$ above for Th1 and Th3, $20 \mathrm{~mm}$ below for Th2 and Th4).

This opening time is in very good agreement with the numerical simulation result $(378 \mathrm{~s})$. That later result combined with the good numerical estimation of the thickness of solid warrants the validity of the simple model we use for the case where convection effects caused by flow recirculation above the cold plug can be neglected.

\section{Extrapolations to MSFR}

The cold plug can be a key component of the MSFR fuel salt draining system which in this reactor concept has to ensure two key safety functions: reactivity control and fuel cooling. The working principle proposed for the cold plug design relies on the control of the heat transfer balance between an electrical heater and a gas cooler. The heat balance established between the heater and the cooler determines whether the salt in the plug region solidifies or melts. The running parameters of these two components leading to the solidification of the molten salt inside the device are the "normal parameters". Disturbance in these parameters (such cooling stopping or overheating of the area) will lead to the salt ingot melting and thus the cold plug opening. Therefore, during nominal operation of the MSFR and as long as the cold plug is cooled down, the salt inside should remain in solid phase. In the event of accidents leading to a total loss of in-site electrical power (such as a Station Blackout) or as result of an intended operator action, the cooling disk will no longer be cooled. The thermal energy stored in the steel mass will then be transferred by conduction to the cooper disk causing the solidified salt region to rapidly melt and the plug to open. The cold plug opening allows for the fuel salt draining from the core cavity by gravity force into the salt draining tanks [5]. Numerical simulations based on a fuel inventory of de $18 \mathrm{~m}^{3}$ of salt to be drained (reactor core + pipes + thermal exchangers) and the draining system geometries shown in Figure 14 from (see SAMOFAR D3.1 report) presents the geometry used for the numerical simulation of the draining. 


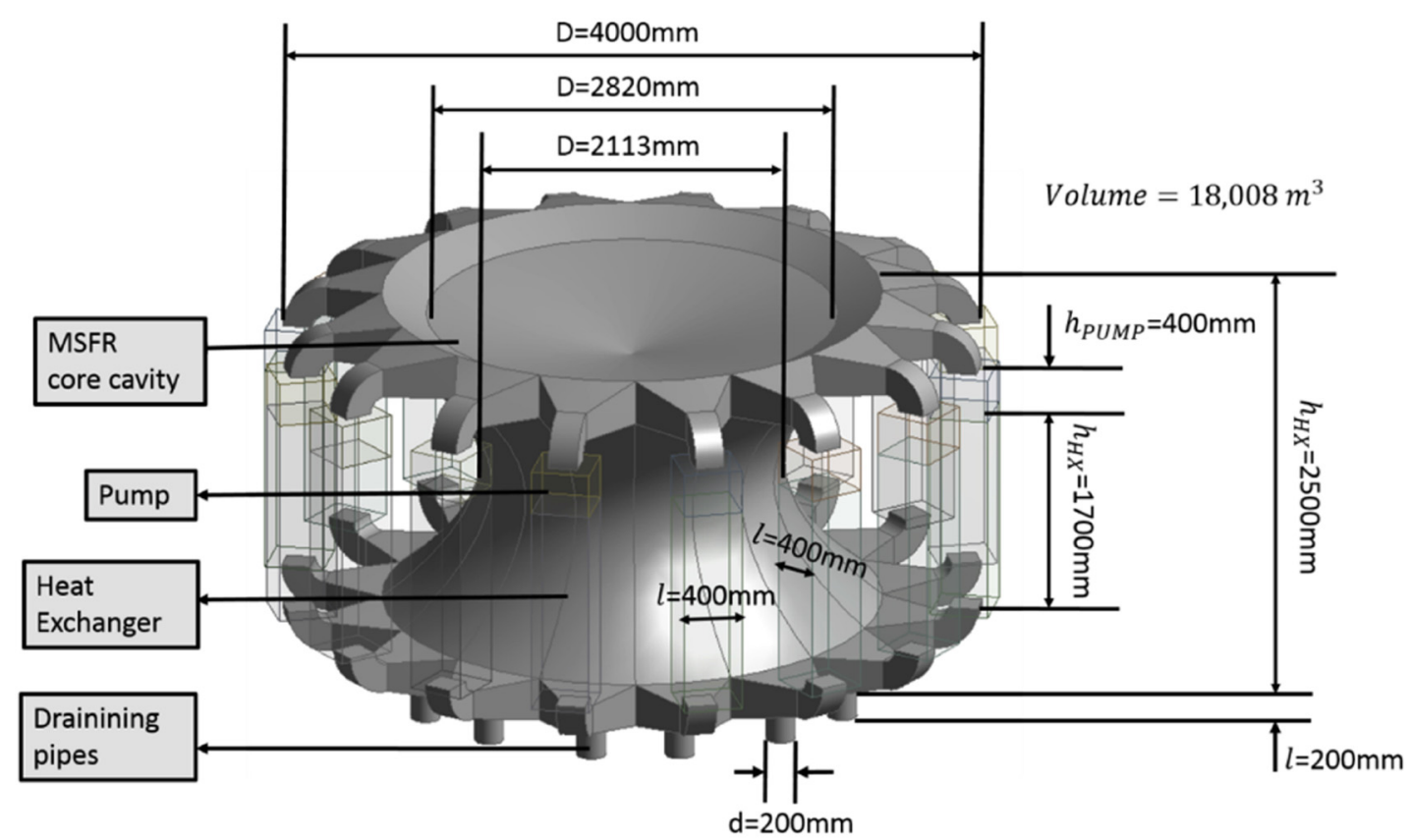

Fig. 14. Geometry of the MSFR fuel circuit for the fuel draining transient studies (SAMOFAR D3.1 report).

This simulation has shown that an adequate behavior can be obtained with draining pipes diameter set to $200 \mathrm{~mm}$. This result has been obtained using a two phase flow thermal-hydraulic model [5] implemented in the CFD code OpenFOAM where the thermal-hydraulic field is solved independently of the nuclear power generation. This supposition is good enough for providing an approximate temperature and velocity fields in the reactor before the draining takes place. The fuel-salt is assumed to be an incompressible, isotropic, Newtonian fluid. Furthermore, the Boussinesq approximation is assumed to be valid over the whole domain. For the conservation of momentum, external body and surface forces, other than gravity, are not considering. For the energy equation, no radiation heat transfer, pressure expansion work or viscous dissipation is considered. Furthermore, no phase change and no presence of bubbles is assumed over the domain. The effects of the covert gas flow during the draining are however considered. Based on these results, a diameter of $200 \mathrm{~mm}$ was adopted for the pipe of the cold plug for extrapolations of our experiments to a reactor component.

During SWATH experiments, 304L stainless steel has been used for pipes and steel mass. Internal diameter was $50 \mathrm{~mm}$. Cooling disk was made of copper. In reactor case, 304L stainless steel has to be replaced by Hastelloy $\mathrm{N}$ or a more suitable alloy if possible. In spite of its very good thermal conductivity copper is not suitable for high temperature use (melting temperature $=1084^{\circ} \mathrm{C}$ ) because of the relative poor mechanical properties.

During the SWATH cold plug studies, the plug formation did not require a downstream valve because the salt level was maintained by the tank pressure control system but in reactor liquid salt has to be introduced and kept in the system during solidification. Then, in a cold plug reactor concept, we add a valve (green part in Fig. 15) to block the molten salt after its filling. This valve will likely have a large size in order to decrease the pressure drop during draining. The valve for salt filling can be a smaller one. In the same way, the gas valve for emptying of the downstream part of the plug after solidification. Of course the chosen components (salts valves and gas valve with their actuators) have been taken only for example to illustrate the occupied volume and the complexity of the system. Other type of valves can be used, but a significant reduction of the volume and complexity should not be expected.

A numerical study on the extrapolation of the cold plug for $\mathrm{LiF}-\mathrm{ThF}_{4}$ salt and to a $200 \mathrm{~mm}$ in-diameter pipe (factor of four compared to the SWATH-S plug) has been done using Hastelloy $\mathrm{N}$ for the thermal mass and pure molybdenum for the cooling disk. The general cooler shape (flower like) of the cooling disk used in the SWATH experiments has been kept but the thickness of the disk has been increased to $30 \mathrm{~mm}$. Data used for the numerical simulation are gathered in Table 2. Both thermal and mechanical numerical simulations have been done. The same thermal numerical model is used as for SWATH.

From mechanical point of view, during normal running of the plug, the hydrostatic pressure supported by the cooling disk in a reactor can be high. The objective of the mechanical simulation is to validate the mechanical resistance of the cold disk supporting the cold plug because a failure in this support will cause an involuntary emptying of the core. For conservatism, we supposed a pressure of 25 bars (hydrostatic pressure and over pressure in core vessel). The 25 bars pressure is apply on one side of the cold disk at the service temperature $\left(600^{\circ} \mathrm{C}\right)$. The material used is molybdenum and the $0.2 \%$ proof stress is around $350 \mathrm{MPa}$ (criteria). Simulations were performed with 


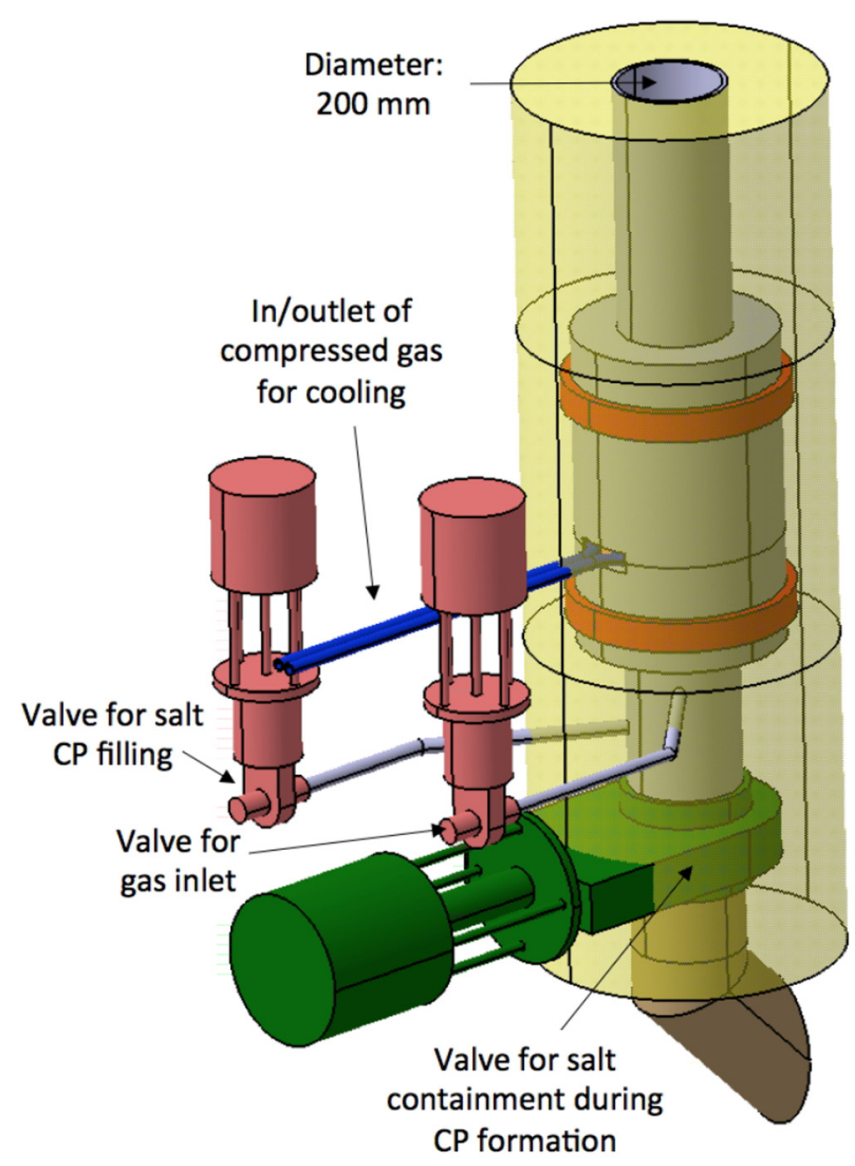

Fig. 15. Main required components of a reactor cold plug.

ANSYS mechanical 19.0 (Linear static solver with small displacement formulation). Simulation results (stress distribution across the disk and elastic deformation of the disk) show that:

- the maximum Tresca stress combination of $159 \mathrm{MPa}$ (bending stress), is inferior to the rupture criterion $(350 \mathrm{MPa})$;

- the 7.8 tons thrust do not create problems whatever the shape of the internal holes in the case of a 200 in-diameter pipe. In case of larger diameter, reinforcement of the disk would be required;

- the mechanical simulation done is valid for closed plug (temperature $\leq 600{ }^{\circ} \mathrm{C}$ ).

Thermal numerical simulations were performed to evaluate the closing time, the cold plug size in normal operation and the opening time. Simulations were performing with ANSYS mechanical 19.0 (Finite Element Method) and with the same model as for SWATH experiments. Only a $22.5^{\circ}$ sector the cold plug is modeled to reduce the simulation time.

The parameters for the experiments and for the numerical simulations are the compressed air volumetric flow rate in the cooling plate and the temperature setup by the heating elements on the steel structure. The used values are given for the different stages described below. These parameters have been chosen for the different stages of the plug reactor formation in order to obtain a $100 \mathrm{~mm}$ total thickness plug in normal operation and a solidification time of $30 \mathrm{~min}$. Other results can be obtained for slightly different conditions but the presented case seems to be a good compromise and allows illustrating the possibility of use for reactor draining.

Stage 1: Cold plug formation time of $30 \mathrm{~min}$ with the following hypothesis:

- upper heater temperature: $610{ }^{\circ} \mathrm{C}$;

- lower heater temperature: $600^{\circ} \mathrm{C}$;

- cooling gas flow rate $45 \mathrm{~m}^{3} \cdot \mathrm{h}^{-1}$;

- filling salt temperature: $650^{\circ} \mathrm{C}$ (see Fig. 16).

After $1800 \mathrm{~s}$, a solidified salt mass has growth over the contact region between the salt an the cooling disk and the steel masses walls due to the lower temperatures close to the metallic walls. At this time, the numerical simulation predicts that the plug is closed but it has a very different shape than the one at the final state during normal operation (see the following stage). The numerical simulation predicts that the solidification process would require a few hours before the solidified plug reaches the final size (steady state).

Stage 2: Normal operation service of a $100 \mathrm{~mm}$ thick plug with the following hypothesis:

- upper heater temperature: $670^{\circ} \mathrm{C}$;

- lower heater temperature: $660^{\circ} \mathrm{C}$;

- cooling gas flow rate $13 \mathrm{~m}^{3} \cdot \mathrm{h}^{-1}$ (see Fig. 17).

Figure 17 presents the final steady state shape of the normal cold plug. The shape of the plug has progressively changed to reach that of balance conditions in normal operation. The temperature of the heater is higher than for the stage 1, and the cooling flow rate lower. From the SWATH cold plug experiments we know that the stabilization from the closing moment to the cold plug normal shape for a service operation can take at least five hours. In the reactor case that time can be much longer.

Stage 3: Cold plug opening with the following hypothesis: - upper heater temperature: $670^{\circ} \mathrm{C}$;

- lower heater temperature: $660^{\circ} \mathrm{C}$;

- cooling gas flow rate $13 \mathrm{~m}^{3} \cdot \mathrm{h}^{-1}$ (see Fig. 18).

The initial parameters are the same as those of stage 2 . At the zero time $(t=0 \mathrm{~s})$ heating and cooling of the plug are simultaneously stopped and the system evolves on its own thermal inertia. The appearance of a liquid path for the salt occurs at about $t=401 \mathrm{~s}$.

In all numerical simulations convection in the liquid part above the plug is not taken into account. It will depend on the position of the cold plug against the reactor core. If the plug is too close to the core, high speed salt flow in the core can create convection in the pipe above the plug and led to thermal modifications in the area around the plug. Then, parameters (cooling and heating) used to stabilize the plug in normal condition have to be changed. The design of the core area above the plug would then greatly influence the stability and the parameter of the plug. These phenomena has been identified during experiments done in 
Table 2. Data used for the reactor cold plug simulation.

\begin{tabular}{lllll}
\hline & Temperature & Hastelloy $\mathrm{N}$ & Molybdenum & $\mathrm{LiF}^{-\mathrm{ThF}_{4}}$ \\
\hline Thermal conductivity $\left(\mathrm{W} \cdot \mathrm{m}^{-1} \cdot \mathrm{K}^{-1}\right)$ & 600 & 20.3 & 126 & 1 \\
Density $\left(\mathrm{Kg} \cdot \mathrm{m}^{-3}\right)$ & 600 & 8860 & 10200 & 4100 \\
Heat capacity $\left(\mathrm{J} \cdot \mathrm{Kg}^{-1} \cdot \mathrm{K}^{-1}\right)$ & 600 & 565 & 280 & 900 \\
Latent heat $\left(\mathrm{J} \cdot \mathrm{Kg}^{-1}\right)$ & & & & $1.59 \times 10^{5}$ \\
Melting temperature $\left({ }^{\circ} \mathrm{C}\right)$ & & 1300 & 2600 & \\
\hline
\end{tabular}

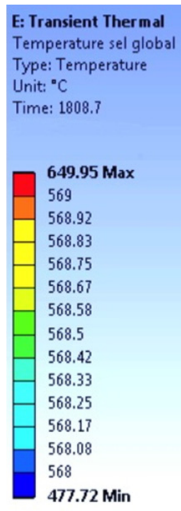

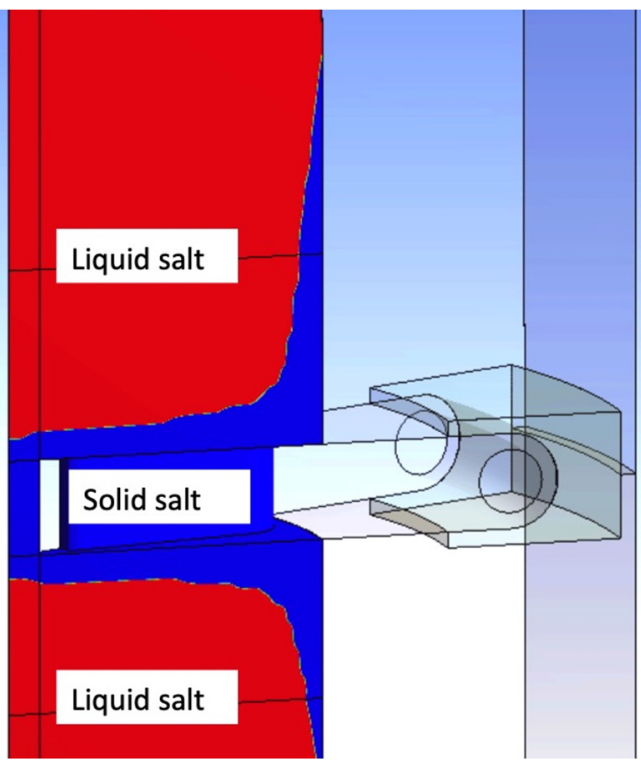

Fig. 16. Cold plug formation stage.

the FFFER loop, where thermal conditions used for static studies in and horizontal plug did not permit to keep the plug in place when dynamic conditions were imposed near the upstream pipe. In a reactor case, it is not realistic to predict all situations able to appear notably in transient conditions. The recommendation is to put the plug system sufficiently away from the core and use a triggering system (based on the core temperature detection for example) to start the opening of the plug by shutting down the cooling and heating power.

\section{Conclusions and perspectives}

The cold plug is a key safety component of the fuel draining system in MSR. An overview of a cold plug design for molten salt applications has been presented. Two set of experiments have been discussed: FFFER loop and SWATH. Design improvements introduced in each of these experiments have been described. These improvements include the use of a cooper plate to enhance the heat balance control in the plug and thus allow for a more stable and fast operation. Holes shape and size have also been optimizing based of the experimental data. Comparison between numerical simulations and the experimental data show a reasonable agreement. Next design steps include the

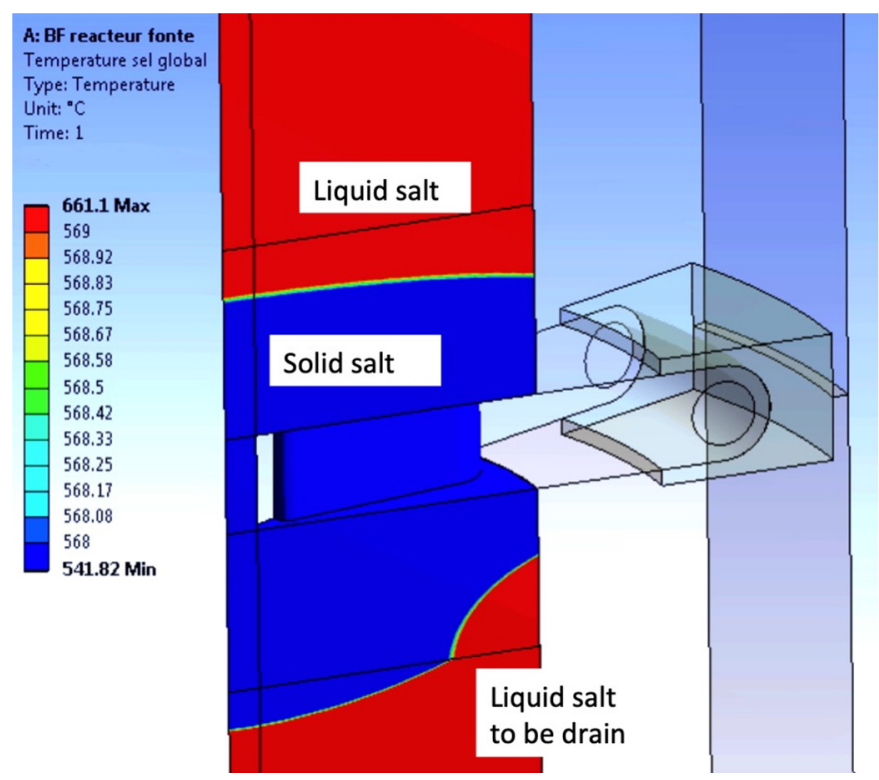

Fig. 17. Closed cold Plug in normal operation.

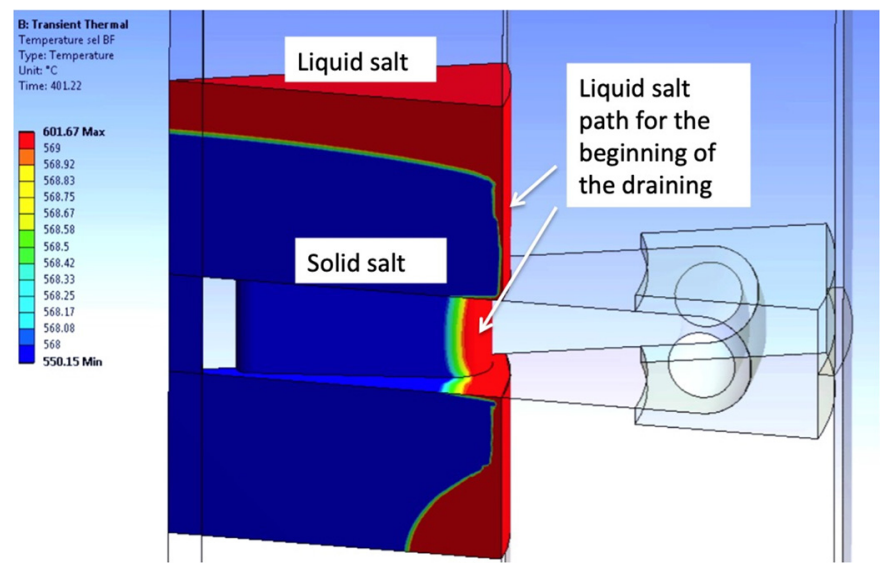

Fig. 18. Opening of the plug, the part of the plug in contact with the wall is at liquid state. The picture corresponds to a time of $400 \mathrm{~s}$ from the cooling and heating stop.

development of more accurate models including recent advances in solidification modeling for a eutectic salt [6].

SWATH project has received funding from the Euratom research and training program 2014-2018 under grant agreement No 661891 . The content of this article does not reflect the official 
opinion of the European Union. Responsibility for the information and/or views expressed in the article lies entirely with the authors. The authors would also thank you the Carnot EnergieGrenoble and the CNRS-IN2P3 for the funding provided for the previous FFFER experiment.

\section{Author contribution statement}

Julien Giraud is a research Engineer at CNRS with specialization on thermo-mechanical works both from point of view of design and implementation. He conducted the whole SWATH facilities design, construction and operation and the numerical simulations related to the cold plug.

Veronique GHETTA is a researcher at CNRS with specialization in physico-chemistry of materials, interfacial properties of systems involving liquid-solid-gas phases. She is in charge of the experimental research using molten salt at LPSC Grenoble. She participated in the definition of the SWATH project and in the construction and operation of the facilities.

Pablo Rubiolo is a professor at the Grenoble Institute of Technology. He works as a reactor physics researcher at LPSC Grenoble, with specialization on advanced nuclear reactor design, nuclear fuel and nuclear core multiphysics numerical modeling. He participated in the definition of the SWATH project, in the choice of the experiments and in the numerical studies on the reactor concept.

Mauricio Tano Retamales is a $\mathrm{PhD}$ student involved in numerical simulations on multi-physics for new nuclear concepts using molten salt. He participated in the design of experimental sections of SWATH and in the reactor draining numerical simulations.

\section{References}

1. M. Richardson, Development of freeze valve for use in the MSRE, ORNL-TM-128, Oak Ridge National Laboratory Report, 1962

2. M. Richardson, Freeze valves, MSRE systems and components performance, ORNL-TM-3039, Oak Ridge National Laboratory Report, pp. 341-355, 1973

3. D. Heuer, E. Merle-Lucotte, M. Allibert, M. Brovchenko, V. Ghetta, P. Rubiolo, Towards the thorium fuel cycle with molten salt fast reactors, Ann. Nucl. Energy 64, 421 (2014)

4. P.R. Rubiolo, M. Tano Retamales, V. Ghetta, J. Giraud, High temperature thermal hydraulics modeling of a molten salt: Application to a molten salt fast reactor (MSFR), ESAIM: Proc. Surv. 58, 98 (2017)

5. M. Tano-Retamales, P. Rubiolo, J. Giraud, V. Ghetta, Multiphysics study of the draining transient in the molten salt fast reactor, in 2018 International Congress on Advances in Nuclear Power Plants (ICAPP 18)

6. M. Tano-Retamales, P. Rubiolo, O. Doche, Progress in modeling solidification in molten salt coolants, Model. Simul. Mater. Sci. Eng. 25, 7 (2017)

Cite this article as: Julien Giraud, Veronique Ghetta, Pablo Rubiolo, Mauricio Tano Retamales, Development of a cold plug valve with fluoride salt, EPJ Nuclear Sci. Technol. 5, 9 (2019) 